\title{
An Optimized NMR Stripline for Sensitive Supercritical Fluid Chromatography-Nuclear Magnetic Resonance of Microliter Sample Volumes
}

\author{
Sebastiaan van Meerten, ${ }^{*}$ Fleur van Zelst, Koen Tijssen, and Arno Kentgens* \\ Cite This: Anal. Chem. 2020, 92, 13010-13016 \\ Read Online
}

ACCESS 1

山 Metrics \& More

回国 Article Recommendations

Supporting Information

ABSTRACT: To optimize sensitivity, there has been an increasing interest in the miniaturization of NMR detectors. In our lab, a stripline NMR detector has been developed, which provides high resolution and is scalable to a large range of sample volumes. These features make it an ideal detector for hyphenated techniques. In this manuscript, we demonstrate a stripline probe, which is designed for combining supercritical fluid chromatography (SFC) experiments with NMR. It features a novel stripline chip, designed to reduce the signal from the contact pads, which results in an improved lineshape. An external lock circuit provides stability over time to perform signal averaging or multidimensional experiments. As proof of concept, we demonstrate the SFC-NMR technique with this stripline probe using a mixture of cholesterol
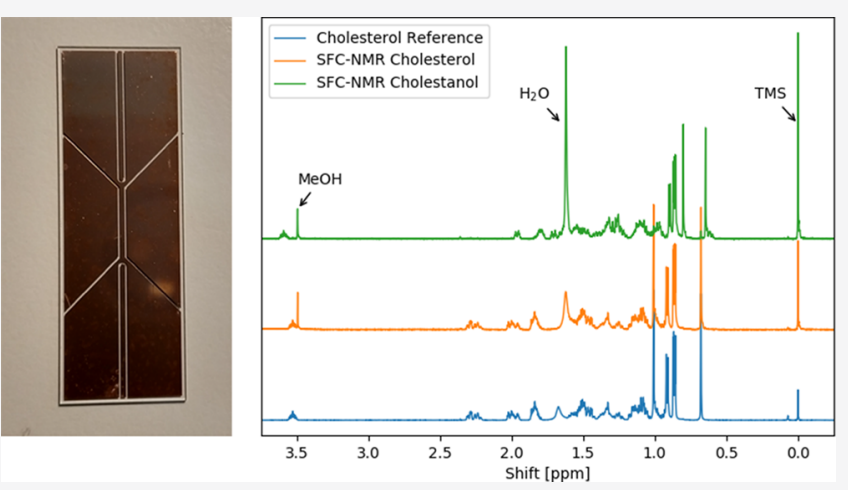
and cholestanol, which is relevant for studying cerebrotendinous xanthomatosis. Additionally, this probe makes it possible to record high-resolution spectra of samples with a high spin density. This means that it is possible to directly observe shifts due to the nuclear demagnetizing field in the "homomolecular" case, which is challenging using conventional probes due to broadening effects from radiation damping.

\section{INTRODUCTION}

In recent years, there has been an increasing interest in miniaturizing detectors for nuclear magnetic resonance (NMR) spectroscopy. ${ }^{1}$ This is because smaller coils can provide a higher sensitivity per spin for mass-limited samples. For example, for a flat circular coil, the sensitivity per sample volume is inversely proportional to its diameter, assuming the resistance of the coil does not change. Decreasing the coil diameter by a factor two doubles the signal-to-noise ratio of the NMR spectrum. Although the sensitivity increases, the number of spins that will fit inside the detected volume decreases. The size of the detector should therefore be matched to the available sample volume for optimal sensitivity. For mass- or volume-limited samples, such as body fluids, it is worthwhile to use a miniaturized detector. The sensitivity of the probe not only depends on its size but also on the coil design. Most of the research has been focused on developing planar helical coils and tightly wound solenoid microcoils. ${ }^{2-5}$ An alternative design is the planar microslot waveguide probe. ${ }^{6}$

However, with these types of microcoils, the resolution is often significantly reduced due to the close proximity of the microcoil structure to the sample. In our lab, a stripline detector has been developed, ${ }^{7}$ which solves this issue by using a planar structure oriented along the main magnetic field. The stripline NMR detector can record spectra with high sensitivity of samples contained within fused-silica capillaries. Also, the stripline can easily be designed for a wide range of sample volumes. This makes it straightforward to hyphenate this type of NMR detector to other techniques such as chromatography and capillary electrophoresis. ${ }^{8}$ An interesting development is the hyphenation of supercritical fluid chromatography (SFC) with stripline NMR., ${ }^{9,10}$

In this manuscript, we demonstrate a novel NMR probe design, which is optimized for on-line SFC-NMR experiments. It features an improved stripline design, which provides a further increase in resolution, and an external lock circuit to provide stability over time for longer experiments. An interesting application of SFC-NMR is the separation and identification of lipids. In the work by Oostendorp et al., ${ }^{11}$ the analysis and quantification of lipids in body fluids are performed using ${ }^{1} \mathrm{H}$ NMR without separation in a regular NMR setup. However, when too many lipids are present or when some are present at high concentration, identifying and

Received: April 28, 2020

Accepted: August 31, 2020

Published: August 31, 2020 
quantifying the lipids can be difficult in these crowded NMR spectra. SFC- ${ }^{1} \mathrm{H}$ NMR could resolve this problem since the lipids are separated before NMR analysis, thus solving the issue of a spectral overlap. We demonstrate the use of this novel probe design in a SFC-NMR setup for the separation and structural analysis of a mixture of cholesterol and cholestanol. This can be relevant for analyzing the blood plasma of patients suffering from cerebrotendinous xanthomatosis (CTX), which shows an accumulation of cholestanol due to a defect in a gene coding for the enzyme sterol 27-hydroxylase.

\section{EXPERIMENTAL SECTION}

Split-Contact Stripline. Although the stripline detector has a good homogeneity compared to other microcoil designs, the resonances often exhibited a broadening at the base. This is caused by the signal originating from the sample located on the contact pads (the top and bottom parts of the chip shown in Figure 1a). The magnetic field inhomogeneity in these areas is

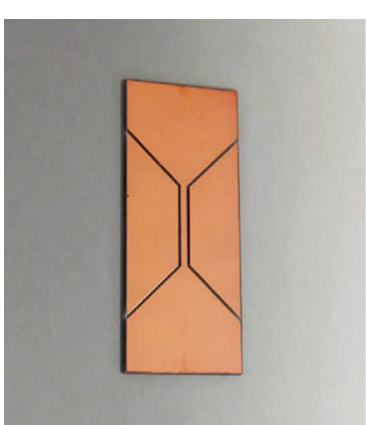

(a) Regular stripline

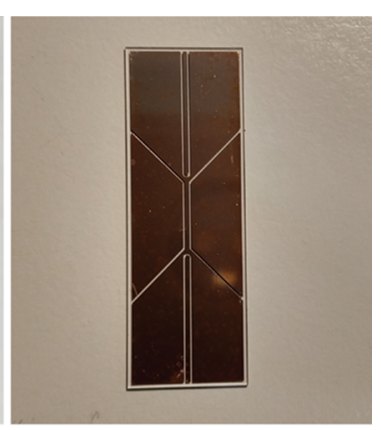

(b) Split-contact stripline
Figure 1. (a) Pictures of the regular stripline and (b) the new splitcontact design. Both designs use ground planes (not shown) to confine the RF field. More information on the stripline design is provided in the Supplementary Information.

caused by the electrical contacts and the casing, which holds the stripline. This means that the magnetic field at the center of the stripline and the contact pads cannot be simultaneously homogenized using shims. To reduce this effect and thus improve the resolution, we have developed a new stripline design where the part of the contact pad directly below the capillary is disconnected from the rest of the contact pad. This reduces the current density near the capillary and thus decreases the RF field strength and sensitivity for the sample located on the contact pads. The design is called the splitcontact stripline and is shown in Figure 1b. To study the behavior of the split-contact stripline, we have used a stripline chip, which is $25 \mathrm{~mm}$ long and $10 \mathrm{~mm}$ wide. The sensitive region of the strip has a width of $500 \mu \mathrm{m}$, separated by gaps of $250 \mu \mathrm{m}$, and the tapered regions have $45^{\circ}$ angles.

The expected difference in $B_{1}$-field strengths between the regular and split-contact stripline was simulated (COMSOL Multiphysics). The details of this simulation are provided in the Supplementary Information. In Figure 2, the simulated $B_{1}$ field strengths (at $600 \mathrm{MHz}$ ) are shown as a function of the position along the stripline $z$. At each position along $z$, the distribution in $B_{1}$-fields is determined in a cylindrical volume at the position of the sample. In blue, the $B_{1}$-profile of a regular stripline is shown and in red the $B_{1}$-profile of the split-contact stripline. At the narrow region of the stripline, there is a distribution in $B_{1}$-field strengths over the capillary. In this

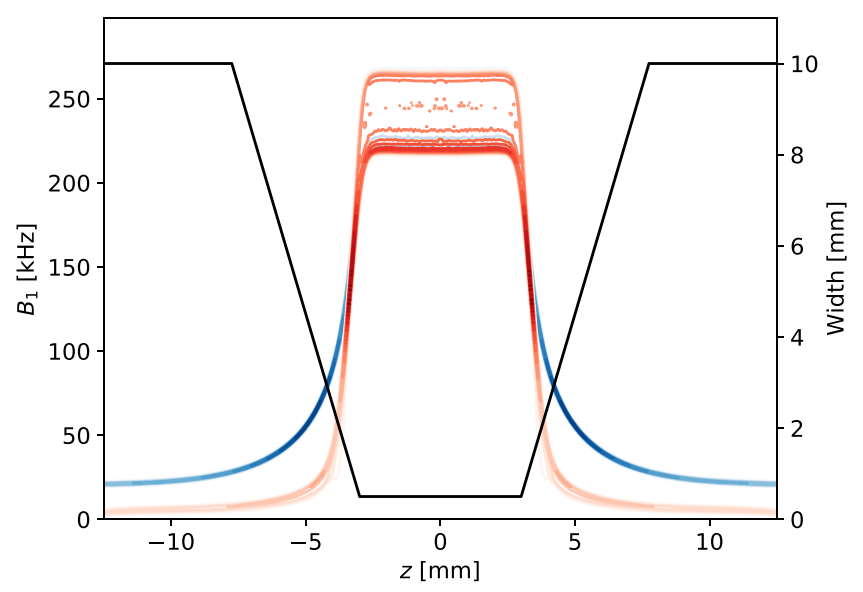

Figure 2. Simulation of the distribution in $B_{1}$-field strengths in the sample as a function of position along the stripline for a regular stripline (blue contours) and a split-contact stripline (red contours). The black line shows the full width of the strip.

region, both profiles are nearly identical; this means that the split contacts hardly influence the main sensitive region of the stripline. On the other hand, outside of the narrow sensitive region, the $B_{1}$-field drops more rapidly in the split-contact case and is approximately 4 times lower compared to the regular stripline. This greatly reduces the excitation and detection of signals originating from sample located outside the narrow region of the stripline.

External Lock. To compensate for drifting of the magnet, most commercial NMR probes contain a deuterium lock channel. In these cases, the deuterium resonance of the deuterated solvent is used to correct for variations in the magnetic field. However, in SFC-NMR, the samples are not always mixed with deuterated solvent. This means that internal locking can only be used in a limit number of cases. For this reason, we have implemented an external lock into the probe design. The external lock circuit consists of a coil wound around a glass tube filled with deuterated solvent, as can be seen in Figure S9, which is part of a resonant circuit that matches the deuterium frequency $(92 \mathrm{MHz})$. In this case, deuterated acetone was used as a lock sample, as its resonance is nearly temperature independent. The lock circuit in this probe design is completely independent of the NMR circuits.

SFC-NMR. The SFC-NMR setup used for this research has been introduced in a previous publication. ${ }^{9}$ It consists of a Waters Acquity $\mathrm{UPC}^{2}$ instrument, which is coupled to a Varian VNMRS spectrometer operating at $600 \mathrm{MHz}$ Larmor frequency $(14.1 \mathrm{~T})$. Selection and concentration of the fraction of interest for NMR analysis proceed as follows; During chromatography, when a peak of interest passes the UV detector, a valve is switched, letting the sample including mobile phase into a purged $100 \mu \mathrm{L}$ sample loop. When the valve switches back, the sample expands to near atmospheric pressure through a fixed restriction in the flowline. The pressure drop causes the $\mathrm{CO}_{2}$ to evaporate and the compound of interest to stay behind on the walls of the tube. This selection procedure can be performed multiple times to collect the sample from multiple chromatographic runs. After selecting the peak, a water flow is switched on, which moves through the loop toward the NMR probe in a PEEK tubing with an inner diameter of $0.254 \mathrm{~mm}$ at a flow rate of $0.1 \mathrm{~mL} \mathrm{~min}^{-1}$. After the tubing is completely filled with water, a plug of strong solvent, 
in this case, $15 \mu \mathrm{L}$ of $\mathrm{CDCl}_{3}$ (Cambridge Isotope Laboratories, 99.8 deuterated $+0.05 \mathrm{v} / \mathrm{v}$ TMS), is introduced into the water flow to elute the sample. This plug flows toward the middle of the NMR stripline detector at which point the flow is stopped. Stopping the flow enables the acquisition of multiple scans to improve the signal-to-noise ratio. After the experiment, the valve returns to its original position and the sample flows back from the NMR probe to the waste. More details on the chromatography used in the SFC-NMR experiments are provided in the Supplementary Information.

For the NMR experiments, a home-built probe with the split-contact stripline as described above was used. The stripline chip used for SFC-NMR is of a larger design (dimensions are provided in the Supplementary Information) to accommodate a capillary with an outer diameter of $700 \mu \mathrm{m}$ and an inner diameter of $500 \mu \mathrm{m}$, which means it has an active detection volume of $\sim 1.6 \mu \mathrm{L}$. The SFC-NMR spectra and reference cholesterol spectrum were acquired with a relaxation delay of $10 \mathrm{~s}$ and an acquisition time of $1 \mathrm{~s}$. For the SFC-NMR spectra, 5250 scans were recorded ( $16 \mathrm{~h}$ and $2 \mathrm{~min}$ ) and 4500 scans for the reference cholesterol spectrum $(13 \mathrm{~h}$ and $48 \mathrm{~min}$, $25.9 \mathrm{mM}$ ). All spectra were recorded with a receiver bandwidth of $8 \mathrm{kHz}$. The spectra are referenced to TMS. The spectra were recorded in blocks of five scans. Before the summation of the spectra, the maxima of the TMS peaks was aligned to compensate for any remaining drift in the spectra over time, and the spectra were baseline corrected. All spectra were processed using ssNake. ${ }^{12}$

\section{RESULTS}

Split-Contact Stripline. The split-contact stripline design was tested by comparing the RF-field strengths with a regular stripline design. For both designs, a chip was fabricated with a constriction length of $6.0 \mathrm{~mm}$ and a width of $500 \mu \mathrm{m}$. Using a capillary (OD $350 \mu \mathrm{m}$, ID $250 \mu \mathrm{m}$ ) with a $1 \mathrm{~mm}$ plug of water in FC-40, the power levels where calibrated such that the constricted part of the stripline chip produced an RF field of approximately $250 \mathrm{kHz}$. Afterward, the water sample was repositioned to the contact pad, and a nutation experiment was performed to determine the $B_{1}$-field produced at the contact pad. The positions of the sample are shown in Figure 3. The repositioning of the capillary did not influence the tuning and matching of the probe. The results of these experiments are shown in Figure 4 . These $B_{1}$-fields correspond well to those predicted by the simulations. The regular stripline produced a

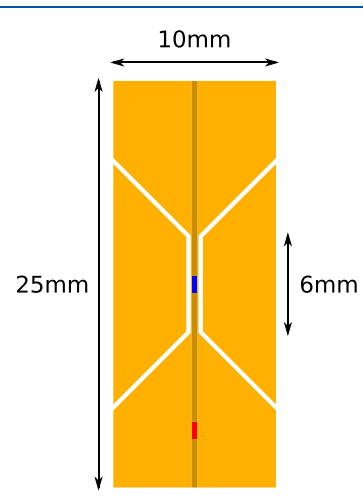

Figure 3. Positions of a sample plug (water in FC-40). The position in blue is used to calibrate the RF field. The position, shown in red, is used to determine the RF-field at the contact-pads.
$B_{1}$-field of approximately $21.5 \mathrm{kHz}$ (simulated: $23.0 \mathrm{kHz}$ ) at the contact pad, whereas the split-contact stripline produced a $B_{1}$-field of only $6.0 \mathrm{kHz}$ (simulated: $6.1 \mathrm{kHz}$ ). As can be seen from the figures, the sensitivity at the contact pads is also significantly lower for the split-contact design, as is expected from the reciprocity principle. ${ }^{13}$ The reduced $B_{1}$-field strength also means that when a pulse with a flip angle of $90^{\circ}$ is given at the center of the stripline; the spins on the split-contacts experience a pulse with a flip-angle of $1.7^{\circ}$ (compared to a flipangle of $7.8^{\circ}$ for a conventional stripline).

The reduced sensitivity of the contacts of the stripline results in an improvement of the resolution. This can be seen in Figure 5 where the $\mathrm{CH}_{3}$ resonance of toluene is compared between a spectrum obtained using regular and split-contact striplines. The FWHM improves from $5.5 \mathrm{~Hz}$ to about $3 \mathrm{~Hz}$; however, the biggest improvement can be seen in the base of the peak where most of the signal originates from the contact pads. The split-contact spectrum is still slightly asymmetric, which is most likely caused by local field disturbances, which are hard to correct using a regular shim system. An example of a source of these local field disturbances is the magnetic impurities in the "seed layer" used in the manufacturing of the stripline chip. One way to compensate for these local field disturbances would be to implement a Shim-on-Chip system. ${ }^{14}$

External Lock. To demonstrate the stability of the external lock with the stripline detector, we have recorded a series of single-pulse NMR spectra over time. In Figure S10, the drift of the $\mathrm{CH}_{3}$ resonance of methanol is shown for a period up to 35 h. What can be observed is that without a lock, the magnetic field takes nearly $10 \mathrm{~h}$ to stabilize completely after the probe has been inserted into the magnet. Furthermore, there are sharp features visible at around 5,15 , and $30 \mathrm{~h}$ after the experiment was started. These are caused by the usage of the Bitter magnets in the High Field Magnet Laboratory (HFML) building about $100 \mathrm{~m}$ away from the NMR lab. To correct for these effects and for the drifting of the magnet, an external lock was implemented in the stripline probe. In this case, the external lock circuit contains a sample of deuterated acetone. Spectra of a sample of acetone were recorded for nearly $30 \mathrm{~h}$. With the external lock, the initial drift after inserting the probe is corrected, as well as the influences of the magnets of the HFML. Some small variations in the resonance frequency can still be seen, with a maximum deviation of about $1 \mathrm{~Hz}$. These variations are most likely caused by slight differences between the sample and lock sample.

Demagnetizing Field. An interesting effect that can be directly observed with this stripline probe is the shift of a resonance due to the nuclear demagnetizing field (long-range dipole-dipole interactions). The nonlinear effects of the nuclear demagnetizing field have been known for a long time, in particular, in the context of paramagnetic impurities, ${ }^{15,16}$ and the multiple echoes caused by the demagnetizing field have been observed experimentally in different materials. ${ }^{17}$

In this experiment, we placed a capillary with an inner diameter of $50 \mu \mathrm{m}$ filled with $\mathrm{H}_{2} \mathrm{O}$ in the stripline probe. The narrow diameter of the capillary minimizes radiation damping and lowers the $B_{1}$-gradient over the sample. Simple one-pulse NMR experiments were performed on this sample with varying pulse widths. The results are presented in Figure 6, which shows that there is a difference in the resonance frequency after a small flip angle compared to a near $180^{\circ}$ pulse. This difference in frequency is about $2.3 \mathrm{~Hz}(3.8 \mathrm{ppb})$. 


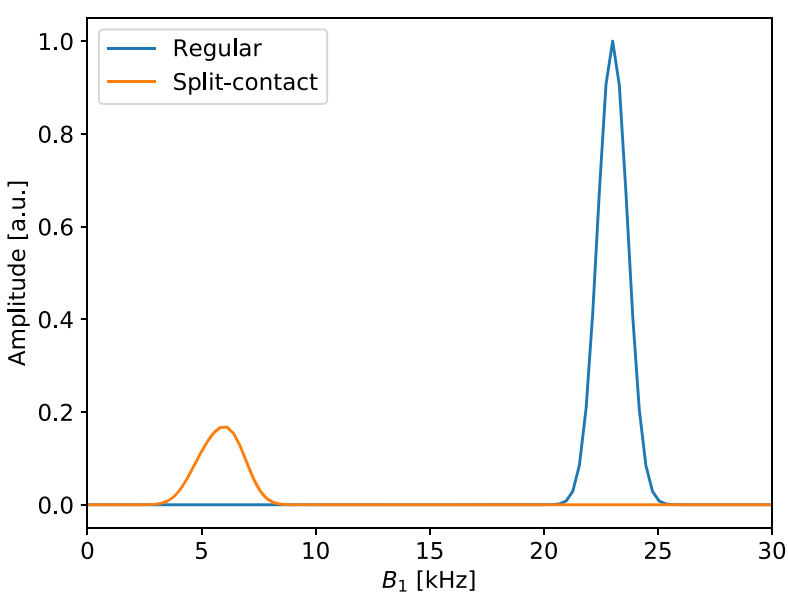

(a) Simulation

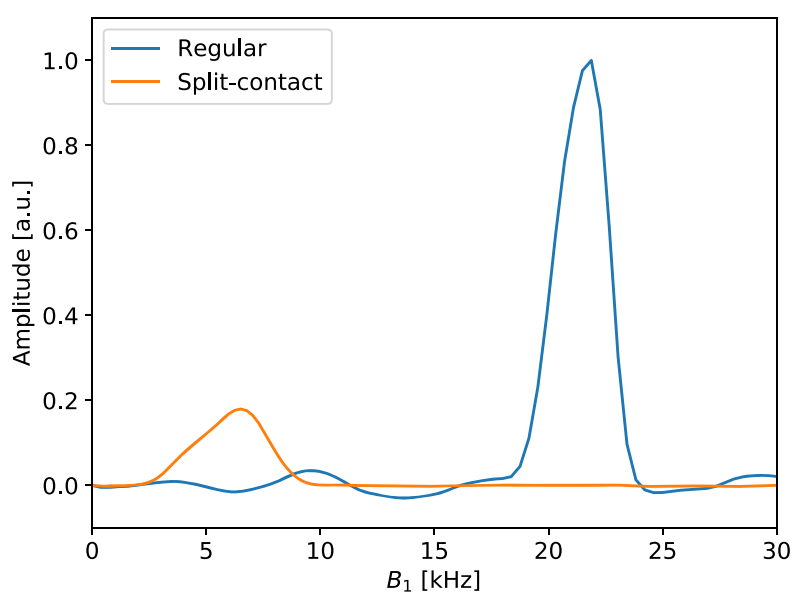

(b) Experiment

Figure 4. (a) Simulation and (b) experiment of the nutation frequency of a $1 \mathrm{~mm}$ plug of water in FC-40. The plug is positioned at the contact pads of a regular stripline (blue) and a split-contact stripline (orange).

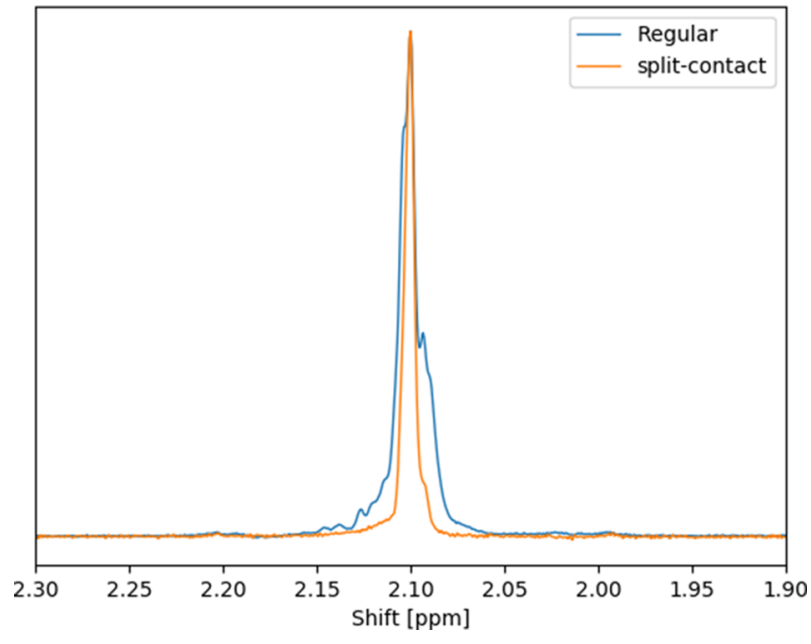

Figure 5. $\mathrm{CH}_{3}$ resonance of toluene recorded using a regular (blue) and a split-contact stripline.

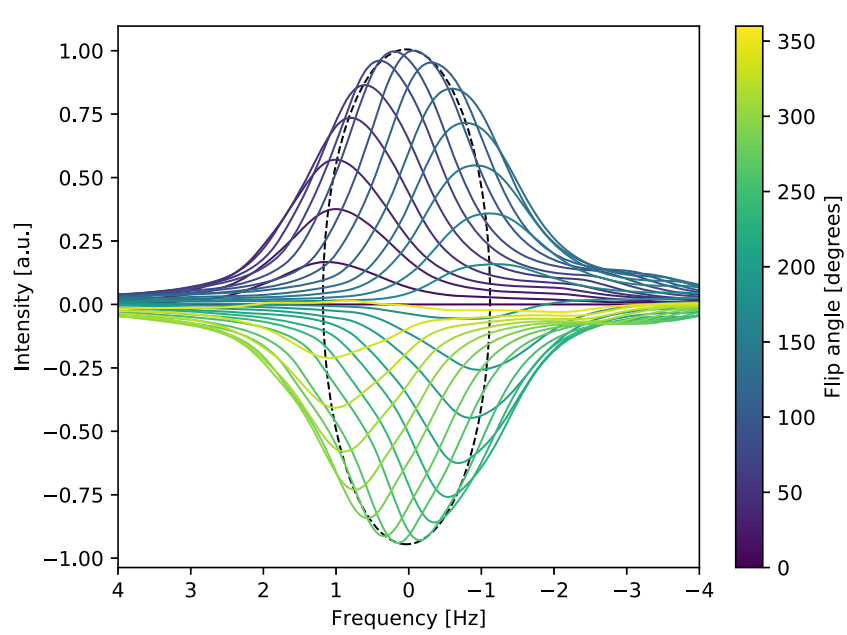

Figure 6. Spectra of $\mathrm{H}_{2} \mathrm{O}$ as a function of flip angle showing the shifting resonance. The black ellipse shows the path that the peak maximum/minimum makes. The width of the ellipse is $2.3 \mathrm{~Hz}$.

Due to the small diameter of the sample (i.e., $50 \mu \mathrm{m}$ ), the $B_{1}$ homogeneity is improved, which means that the resonance shift can be monitored for a number of cycles, as demonstrated in Figure 7a. In this figure, the black line approximately follows the resonance frequency. This line has an oscillation frequency $(87 \mathrm{kHz})$ and a decay rate (Gaussian) identical to that of the signal amplitude (the orange line of Figure $7 \mathrm{~b}$ ) and only has a $90^{\circ}$ phase difference. The resonance frequency also slowly shifts to lower frequency during the experiment, this is most likely caused by a slow increase in the sample temperature.

The effect of this demagnetizing field can be observed in this stripline setup because the sample is locked using an external lock circuit. In regular liquid-state NMR, it is common to lock on the deuterated solvent in the sample. This means that the demagnetizing field shift is not directly observed as shown here since the lock signal will be affected by the demagnetizing field also. It can also result in anomalous crosspeaks, for example, in COSY experiments. ${ }^{18}$

It is interesting to note that the magnitude (in $\mathrm{Hz}$ ) of the shift in Figure 6 cannot be explained by the classical picture of bulk magnetization created by the sum of the individual proton spins. The shift between $0^{\circ}$ and $180^{\circ}$ in the classical picture is predicted to be $1.6 \mathrm{~Hz},{ }^{15,18}$ whereas we observe $2.3 \mathrm{~Hz}$. In the quantum mechanical framework, the shift is described by longrange dipole-dipole interactions, which are not averaged by molecular motion. ${ }^{19}$ In this case, the predicted shift is $\frac{3}{2}$ times larger for "like" spins or so-called "homomolecular" spins. ${ }^{20}$ This is because the B-term in the dipolar Hamiltonian contributes to the total shift for these types of spins.

Direct detection of this kind of effect can only be done conveniently in a microliter setting. Recording spectra of samples with high spin densities in regular liquid NMR probes is usually complicated by other nonlinear effects, such as radiation damping. With the stripline probe, it is straightforward to reduce these effects so that the pulse width effect on the resonance position can be observed with high accuracy.

SFC-NMR. The stripline described above was optimized to analyze microliter $(\mu \mathrm{L})$ sample volumes, thus matching the volumes coming from chromatographic techniques such as SFC. Therefore, we implemented the detector in our hyphenated SFC-NMR system. The design specifications of the stripline chip are provided in the Supporting Information. Due to high solubility of lipids in supercritical $\mathrm{CO}_{2}$, which has similar solvent strength as hexane, SFC is used more often in 


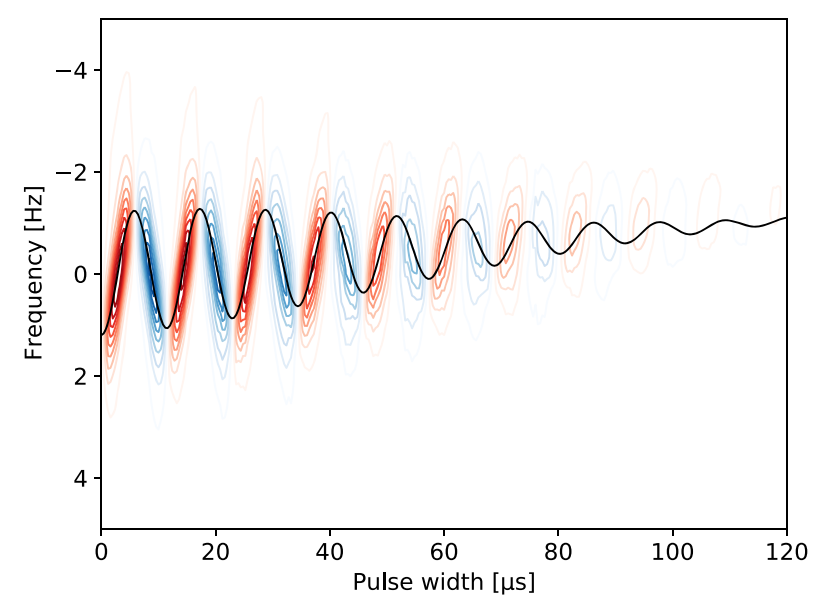

(a) Shift

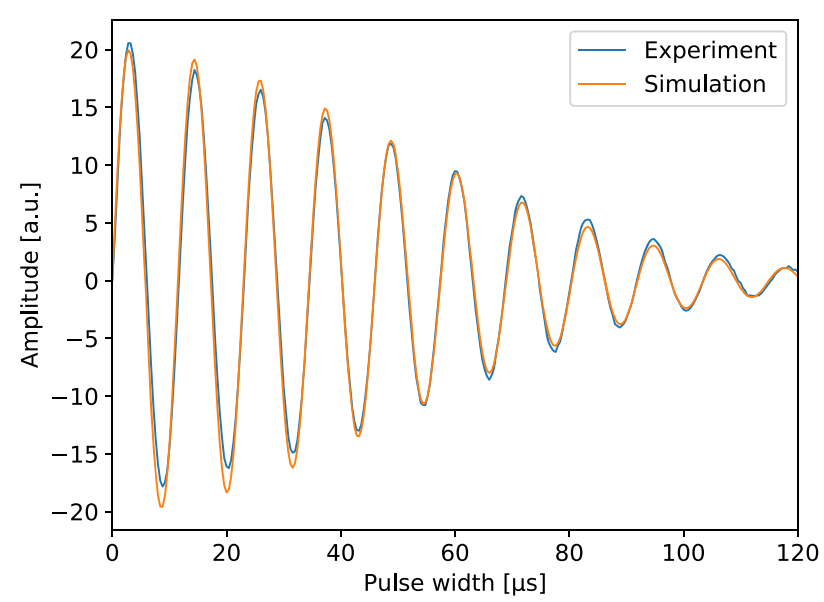

(b) Signal intensity

Figure 7. (a) Shift and (b) amplitude of the proton resonance as a function of RF-pulse width. The black and orange lines have a frequency of 87 $\mathrm{kHz}\left(90^{\circ}\right.$ out of phase) and a Gaussian decay. The black line has an amplitude of $1.2 \mathrm{~Hz}$

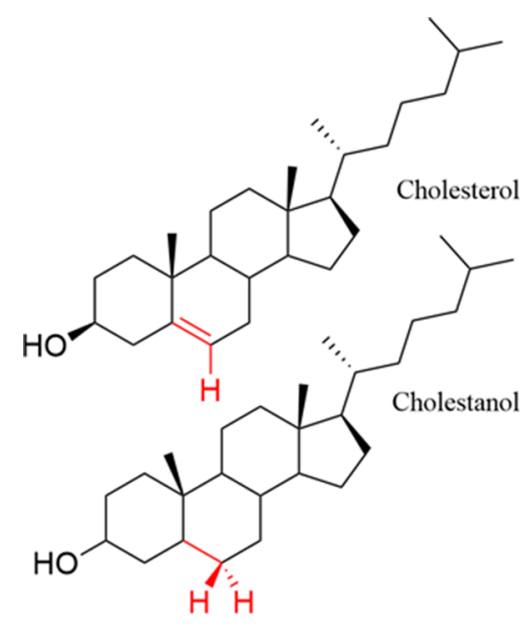
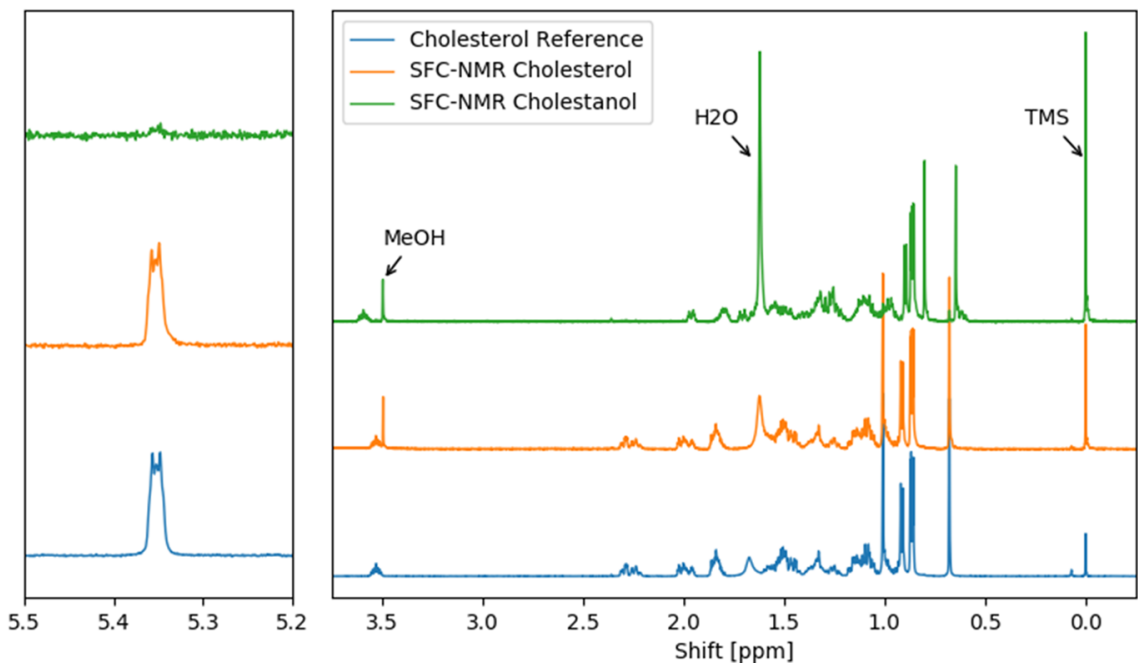

Figure 8. SFC- ${ }^{1} \mathrm{H}$ NMR spectra of the first chromatographic peak (green) and the second chromatographic peak (orange) compared to reference cholestanol ${ }^{1} \mathrm{H}$ NMR spectrum in $\mathrm{CDCl}_{3}$. The molecular structures of cholesterol and cholestanol are shown on the left.

the separation of different classes of lipids in the field of lipidomics. $^{21}$ Here, we look at the SFC separation of cholesterol, one of the most known lipids, and cholestanol, which has a similar structure to cholesterol, differing in only one double bond (Figure 8). For the separation of these compounds, a $1 \mu \mathrm{L}$ mixture of $28 \mathrm{mM}$ cholestanol and $43 \mathrm{mM}$ cholesterol in chloroform was injected into the SFC. The optimal separation conditions were obtained with a Torus Diol column from Waters, using a gradient of $2-5 \%$ methanol in $\mathrm{CO}_{2}$ over $3 \mathrm{~min}$, the result of which can be found in the Supporting information. Although the two molecules are very similar in structure, they can still be separated well under these conditions by SFC.

To test if the hyphenation of SFC-NMR could be a feasible analytic technique in the field of lipidomics, the split-contact stripline detector, optimized for $\mu \mathrm{L}$ volumes, was coupled inline to the SFC apparatus. After SFC separation, both lipids were each collected six times in separate runs, before eluting them to the middle of the stripline NMR detector, in a plug of $15 \mu \mathrm{L}$ of $\mathrm{CDCl}_{3}$. There, the flow was stopped, allowing for the acquisition of multiple scans, to obtain a higher signal-to-noise ratio. To obtain a reference spectrum of cholesterol, instead of selecting the sample from the SFC, a $15 \mu \mathrm{L}$ plug of $26 \mathrm{mM}$ cholesterol in $\mathrm{CDCl}_{3}$ was injected directly into the flow of water toward the NMR spectrometer. The resulting spectra are shown in Figure 8. By comparing the SFC- ${ }^{1} \mathrm{H}$ NMR spectra with reference spectra, it is clear that the first chromatographic peak corresponds to cholestanol and the second peak corresponds to cholesterol. By using the split-contact stripline detector, the resolution and peak shape is improved (compared to using a regular stripline) and peak splittings due to Jcouplings can be observed, e.g., around $3.55 \mathrm{ppm}$. The peak at $5.3 \mathrm{ppm}$, corresponding to the proton on the ring in cholesterol, which is absent in the cholestanol spectrum, confirms the selection of the correct molecules. Also, some shifts are observed for other peaks, reflecting the differences in the molecular structure. A mixture of very similar molecules can thus be separated and characterized by SFC-NMR. If the spectra are examined closely, a small impurity of cholesterol can however be observed in the cholestanol spectrum, probably due to a slight overlap of the peaks during chromatography. This does however not influence the 
structural characterization of the molecule in the spectrum since the concentration of the impurity is very low.

The SFC-NMR spectra shown here were acquired overnight over $16 \mathrm{~h}$, which is a long time for an analysis. The signal-tonoise ratio is, however, much higher than required for the identification of these compounds. A spectrum with a signal-tonoise ratio of 3 for the peak around $5.3 \mathrm{ppm}$ would require an experimental time of only $1 \mathrm{~min}$. This is also due to the advantage of using the stripline probe with a larger volume. In our previous work, ${ }^{9}$ a stripline probe with a detection volume of only $150 \mathrm{~nL}$ was used. The current stripline has a volume of $\sim 1.6 \mu \mathrm{L}$. The volume that is selected from the SFC is $100 \mu \mathrm{L}$ for each selected peak. To get a higher sample concentration in the NMR detector, however, the mobile phase is evaporated out and the sample is redissolved in $15 \mu \mathrm{L}$ of solvent, in this case, deuterated chloroform. The detection volume of $1.6 \mu \mathrm{L}$ therefore matches the sample volume much better than in our previous probe in which only a small part of the sample was used for detection. The current probe volume could be increased even more, to better match the sample volume from the SFC. However, some mixing of the sample plug with the transportation medium, water, occurs at the edges of the plug. To prevent the water from interfering with the spectra, it is best not to use the full sample plug for detection but only the middle part. This $\mu \mathrm{L}$ volume stripline probe is therefore the best match in this hyphenation of SFC with in-line NMR detection. Although the absolute sensitivity of the stripline decreases by scaling up the design, the increased number of spins in the active detection volume leads to an overall gain in signal intensity. As an estimation, to obtain a signal-to-noise ratio of 3 for the cholesterol spectrum with the smaller volume stripline probe, $15 \mathrm{~min}$ of the experimental time is required, instead of $1 \mathrm{~min}$. It is therefore beneficial to match the stripline probe volume to the sample volume obtained from the SFC.

The concentrations of cholestanol and cholesterol used in this SFC-NMR analysis are 28 and $43 \mathrm{mM}$, respectively, which are high compared to the concentrations of 0.2 and $3 \mathrm{mM}$ that are present in the blood plasma of patients suffering from CTX. $^{11}$ If a signal-to-noise of 3 would be taken as the minimum for identification of cholestanol/cholesterol, the concentrations of cholestanol and cholesterol can go down to 0.8 and $1.2 \mathrm{mM}$, respectively. If enough sample is available, more injections from the SFC can also be an option to get a higher concentration for the NMR analysis. In this experiment, the same peak was selected six times, in six separate SFC runs, after which it was eluted in one plug of chloroform to the NMR detector. This was done to obtain a higher concentration of the sample for detection. For the six injections that were done in this analysis, only $12 \mu \mathrm{L}$ of the sample was needed. To get even higher concentrations of the sample into the detector, while keeping the same initial sample concentration going into the SFC, more injections can be done. For example, by selecting the peak 12 times from the SFC, a gain of a factor 2 in concentration is achieved. This either enables a shorter analysis time in NMR, gaining a factor 4 in time, or this allows going to lower initial sample concentrations of 0.4 and $0.6 \mathrm{mM}$ for cholestanol and cholesterol, respectively. This then approaches the concentrations that are present in patients suffering from CTX, making SFC-NMR a feasible technique for detecting this biomarker.

\section{CONCLUSIONS}

In this manuscript, we presented a stripline probe optimized for (in-line) detection of $\mu \mathrm{L}$ sample volumes and showed a proof-of-concept application of SFC-NMR. The stripline has an active volume of approximately $1.6 \mu \mathrm{L}$. A novel design minimizes the signal from the contact pads, which results in an improved lineshape compared to the traditional stripline. An external lock circuit provides long term stability for multidimensional experiments and signal averaging. This high resolution and stability make it possible to record spectra of small sample volumes with high spin densities. This way, it was possible to directly observe the shift due to the distant dipolar field. To our knowledge, a direct observation of this effect for "like" spins has not been demonstrated previously under thermal conditions due to resolution limitations.

This type of stripline probe is well suited for hyphenated chromatography-NMR systems. The stripline described above was optimized to analyze $\mu \mathrm{L}$ sample volumes, thus matching the volumes coming from chromatographic techniques such as SFC. We therefore used the optimized stripline detector in our hyphenated SFC-NMR system.

An SFC-NMR setup has been demonstrated to separate a mixture of cholesterol and cholestanol with subsequent identification by NMR. These lipids are present in high concentrations in blood plasma of patients suffering from cerebrotendinous xanthomatosis (CTX). Both compounds can be isolated using this setup and analyzed with good resolution. In this proof-of-principle experiment, concentrations of cholesterol and cholestanol of 43 and $28 \mathrm{mM}$, respectively, were used for the analysis. However, with simple adaptations, like selecting the sample more often, this setup allows for the detection and identification of lipids in sub $\mathrm{mM}$ range, thus approaching the concentrations of $3 \mathrm{mM}$ cholesterol and 0.2 $\mathrm{mM}$ cholestanol that are present in patients suffering from CTX. Although experiments still need to be performed on real patient samples, this is a proof-of-principle that the SFC-NMR setup with the optimized stripline probe can be a feasible analysis technique for full structural characterization of lipids in body fluids.

\section{ASSOCIATED CONTENT}

Supporting Information

The Supporting Information is available free of charge at https://pubs.acs.org/doi/10.1021/acs.analchem.0c01827.

Stripline simulation, SFC-NMR stripline probe, and SFC-NMR (PDF)

\section{AUTHOR INFORMATION}

\section{Corresponding Authors}

Sebastiaan van Meerten - Magnetic Resonance Research Center, Radboud University, Nijmegen 6525 AJ, The Netherlands; Email: b.vanmeerten@science.ru.nl

Arno Kentgens - Magnetic Resonance Research Center, Radboud University, Nijmegen 6525 AJ, The Netherlands; 이이.org/0000-0001-5893-4488; Email: a.kentgens@ nmr.ru.nl

\section{Authors}

Fleur van Zelst - Magnetic Resonance Research Center, Radboud University, Nijmegen 6525 AJ, The Netherlands

Koen Tijssen - Magnetic Resonance Research Center, Radboud University, Nijmegen 6525 AJ, The Netherlands 
Complete contact information is available at:

https://pubs.acs.org/10.1021/acs.analchem.0c01827

\section{Notes}

The authors declare no competing financial interest.

\section{ACKNOWLEDGMENTS}

This research received funding from the Netherlands Organisation for Scientific Research (NWO) in the framework of Technology Area COAST (053.21.115). The authors thank Hans Janssen (RU) for technical support and Dr. Jan van Bentum for proposing a split-contact design. The authors would like to thank Prof. Ron Wevers (Radboud UMC) for providing the cholesterol and cholestanol samples and Prof. Han Gardeniers for producing the stripline chips.

\section{REFERENCES}

(1) Korvink, J. G.; MacKinnon, N.; Badilita, V.; Jouda, M. J. Magn. Reson. 2019, 306, 112-117.

(2) Massin, C.; Vincent, F.; Homsy, A.; Ehrmann, K.; Boero, G.; Besse, P.-A.; Daridon, A.; Verpoorte, E.; de Rooij, N. F.; Popovic, R. S. J. Magn. Reson. 2003, 164, 242-255.

(3) Olson, D. L.; Peck, T. L.; Webb, A. G.; Magin, R. L.; Sweedler, J. V. Science 1995, 270, 1967-1970.

(4) Dechow, J.; Forchel, A.; Lanz, T.; Haase, A. Microelectron. Eng. 2000, 53, 517-519.

(5) Kentgens, A. P. M.; Bart, J.; van Bentum, P. J. M.; Brinkmann, A.; van Eck, E. R. H.; Gardeniers, J. G. E.; Janssen, J. W. G.; Knijn, P.; Vasa, S.; Verkuijlen, M. H. W. J. Chem. Phys. 2008, 128, No. 052202.

(6) Maguire, Y.; Chuang, I. L.; Zhang, S.; Gershenfeld, N. Proc. Natl. Acad. Sci. 2007, 104, 9198-9203.

(7) van Bentum, P. J. M.; Janssen, J. W. G.; Kentgens, A. P. M.; Bart, J.; Gardeniers, J. G. E. J. Magn. Reson. 2007, 189, 104-113.

(8) Falck, D.; Oosthoek-de Vries, A. J.; Kolkman, A.; Lingeman, H.; Honing, M.; Wijmenga, S. S.; Kentgens, A. P. M.; Niessen, W. M. A. Anal. Bioanal. Chem. 2013, 405, 6711-6720.

(9) van Zelst, F. H. M.; van Meerten, S. G. J.; van Bentum, P. J. M.; Kentgens, A. P. M. Anal. Chem. 2018, 90, 10457-10464.

(10) van Zelst, F. H. M.; van Meerten, S. G. J.; Kentgens, A. P. M. Faraday Discuss. 2019, 219.

(11) Oostendorp, M.; Engelke, U. F.; Willemsen, M. A.; Wevers, R.

A. Clin. Chem. 2006, 52, 1395-1405.

(12) van Meerten, S. G. J.; Franssen, W. M. J.; Kentgens, A. P. M. J. Magn. Reson. 2019, 301, 56-66.

(13) Hoult, D. I.; Richards, R. E. J. Magn. Reson. 1976, 24, 71-85.

(14) van Meerten, S. G. J.; van Bentum, P. J. M.; Kentgens, A. P. M.

Anal. Chem. 2018, 90, 10134-10138.

(15) Edzes, H. T. J. Magn. Reson. 1990, 86, 293-303.

(16) Belorizky, E.; Fries, P. H.; Gorecki, W.; Jeannin, M. J. Phys. II 1991, 1, 527-541.

(17) Ardelean, I.; Kimmich, R. Chem. Phys. Lett. 2000, 320, 81-86.

(18) Levitt, M. H. Concepts Magn. Reson. 1996, 8, 77-103.

(19) Bechmann, M.; Müller, N. Annu. Rep. NMR Spectrosc. 2017, 92, 199-226.

(20) Desvaux, H. Prog. Nucl. Magn. Reson. Spectrosc. 2013, 70, 5071.

(21) Yang, Y.; Liang, Y.; Yang, J.; Ye, F.; Zhou, T.; Gongke, L. J. Pharm. Anal. 2019, 9, 1-8. 